\title{
A Common Origin for Organics in Meteorites and Comets: Was It Interstellar?
}

\author{
Conel M. O'D. Alexander \\ DTM, Carnegie Institution of Washington, \\ 5241 Broad Branch, Washington DC 20015, USA \\ email: alexander@dtm.ciw.edu
}

\begin{abstract}
The insoluble organic material preserved in primitive chondritic meteorites shares many similarities with the refractory organic material in interplanetary dust particles and comets, suggesting that there is a genetic link between the organic matter in objects that formed between $\sim 3$ AU and $\sim 30$ AU from the Sun. These similarities include large D and ${ }^{15} \mathrm{~N}$ enrichments in bulk and even more extreme enrichments in isotopic hotspots. The enrichments attest to formation in very cold environments, either in the outer Solar System or the protosolar molecular cloud. There are many properties of this organic material that are consistent with an interstellar origin, but a Solar System origin cannot be ruled out. Similar organic material is presumably an important component of most protoplanetary disks, and heating or sputtering of this material would be a source of PAHs in disks. The soluble organic matter was more heavily effected by processes on the chondritic parent bodies than the insoluble material. Amino acids, for instance, probably formed by reaction of ketones and aldehydes with $\mathrm{NH}_{3}$ and $\mathrm{HCN}$. The accretion of the relatively volatile $\mathrm{NH}_{3}$ and $\mathrm{HCN}$, presumably in ices, strengthens the chondrite-comet connection. However, unlike most comets the water in chondrites, when it was accreted, had $\mathrm{D} / \mathrm{H}$ ratios that were similar to or depleted relative to Earth.
\end{abstract}

Keywords. Comets: general, meteorites, dust, asteroids, Solar System: formation, molecular processes, astrochemistry, astrobiology

\section{Introduction}

The three basic types of extraterrestrial material that are accreted by the Earth and survive atmospheric entry are classified according to size into: interplanetary dust particles (IDPs), micrometeorites (and the related fully melted cosmic spherules) and meteorites. IDPs and micrometeorites almost certainly have both cometary and asteroidal sources, although the proportions of cometary and asteroidal particles remain a matter of debate (Dermott et al. 2002; Nesvorny et al. 2010). The most recent dynamical arguments suggest that most come from Jupiter family comets (Nesvorny et al. 2010). With a few exceptions, those that come from the Moon and Mars, meteorites are fragments of main-belt asteroids (2-4 AU), with a strong bias towards inner belt asteroidal sources (Morbidelli et al. 2002). While there is an overall gradient of spectral classes in the asteroid belt (Gradie, Chapman \& Tedesco 1989), there has been considerable mixing and it is likely that the meteorite collection has sampled many of these classes (Burbine et al. 2002).

Entry velocity, size, density and entry angle together determining the degree of frictional heating that a particle experiences during atmospheric entry and, therefore, the extent to which organics and other temperature sensitive materials are preserved. The IDPs that are collected in the upper atmosphere are small (typically $<50 \mu \mathrm{m}$ ) and low 
density, so that they decelerate relatively gently. Nevertheless, even the least heated of them probably experience temperatures of at least several degrees Celsius for several seconds. Denser particles from the same bodies as the IDPs would probably be destroyed during atmospheric entry, so it is unclear how representative of their parent bodies IDPs are. Micrometeorites (typically 100-1000 $\mu \mathrm{m}$ ) are generally severely heated on atmospheric entry, and so most are unlikely to retain unmodified organic material. However, organics do survive in a rare group of C-rich particles that may have links to comets (Duprat et al. 2010). Evaporation, ablation and poor thermal conduction confine heating to the outer few millimeters of recovered meteorites.

The most primitive meteorites, the chondrites, are composed of materials that formed in the solar nebula or that even predate it. Chondrites can contain up to several weight percent of organic material. The complex suite of compounds that constitute this organic matter can have large $\mathrm{D}$ and ${ }^{15} \mathrm{~N}$ enrichments that are usually interpreted to indicate that they or their precursors formed at very low temperatures. Similar enrichments are seen in IDPs (Messenger 2000; Aléon et al. 2003; Floss et al. 2006; Busemann et al. 2009) and Stardust particles returned from comet Wild 2 (McKeegan et al. 2006; Matrajt et al. 2008). Thus, it seems likely that there is a genetic link between the organics in chondrites and comets. It is still a matter of contention whether the organics formed in the presolar molecular cloud and/or in the early Solar System. A detailed understanding of the compositions of the various components of the chondritic organic matter may ultimately help resolve this debate.

Most chondrites also show evidence of having accreted water, presumably as ice, when they formed. It seems likely that the organics and water ice would have been intimately associated when they accreted, as they are in comets. In which case, the origins of the organics and water may be linked. Understanding the origin of the water in chondrites may also elucidate the origin of the water in the inner Solar System and transport processes in the solar protoplanetary disk (or solar nebula).

\section{Meteorite Classification}

Meteorites are broadly divided into two, the chondrites and achondrites (Krot et al. 2003). Only the most primitive types of meteorite, the chondrites, are generally thought to contain indigenous organic material. This is because achondrite meteorites come from parent bodies that experienced extensive melting, and even wholesale differentiation into silicate mantles and iron cores. Chondrites are comprised of three main components: refractory inclusions, chondrules and fine-grained matrix. Refractory inclusions and chondrules are high temperature $\left(1400-1800^{\circ} \mathrm{C}\right)$ objects that formed in the solar nebula. Organics would not have survived the high formation temperatures of refractory inclusion or chondrule. Consequently, it is only in the matrix that cements the meteorites together that primary organic matter should be found. It is in the matrix that one also finds presolar circumstellar grains that formed around highly evolved stars, such as supernovae and asymptotic giant branch (AGB) stars (Nittler 2003; Alexander 2009), and were inherited from the protosolar molecular cloud.

Historically, the chondrites have been divided into three classes based on their compositions and mineralogies (ordinary, carbonaceous and enstatite). These in turn have been subdivided (Scott \& Krot 2003; Weisberg, McCoy \& Krot 2006) into a number of groups: ordinary chondrites into H, L and LL, carbonaceous chondrites into CI, CM, $\mathrm{CR}, \mathrm{CV}, \mathrm{CO}, \mathrm{CB}, \mathrm{CH}$ and $\mathrm{CK}$, and enstatite chondrites into EH and EL. The name carbonaceous chondrite is a historical one and is a bit misleading since some ordinary and enstatite chondrites contain more $\mathrm{C}$ than some carbonaceous chondrites. The chondrite 
classification scheme is still evolving as more meteorites are found - two new classes ( $\mathrm{R}$ and $\mathrm{K}$ chondrites) have been identified, and a number of individual meteorites do not belong to any recognized group.

The CIs have bulk compositions that are essentially identical to that of the rockforming component (excluding H, He, etc.) of the Sun (Lodders 2003). Because solar (or CI) is the starting composition from which all Solar System materials evolved, the compositional variations of chondrites and their components are generally expressed as deviations relative to CI. The deviations of the compositions of chondrites (and their components) from CI are presumed to be the results of their formation processes in the solar nebula.

After formation, the chondrites experienced secondary modification - protracted episodes of thermal metamorphism and aqueous alteration, and in some cases brief shock heating events - on their parent bodies. A petrographic classification scheme for secondary processes divides the chondrites into 6 types - types 3 to 6 reflect increasing extent of thermal metamorphism, and types 3 to 1 reflect increasing degrees of aqueous alteration. By convention, the chemical classification is followed by the petrologic one (e.g., CI1, CM2, CV3).

\section{Organics in Chondrites}

There have been a number of recent reviews of the organic matter in meteorites and IDPs (Botta \& Bada 2002; Sephton 2002; Gilmour 2003; Sephton 2005; Pizzarello, Cooper \& Flynn 2006). However, with the renewed interest in the subject, the introduction of new techniques, and new samples from Antarctica and the Stardust sample return mission to Comet $81 \mathrm{P} /$ Wild 2, the field is evolving rapidly. Here we focus on the most recent work. The organic matter is normally divided into solvent soluble (SOM) and insoluble (IOM) fractions, and consequently they are discussed separately. The abundances of IOM and the known components of the SOM are summarized in Table 1. Also included in Table 1 are the matrix volume percents because the organic matter would have been accreted by the chondrites in the matrix material.

\subsection{Insoluble Organic Matter}

The IOM shows tremendous variation in its elemental and isotopic compositions both within and between chondrite groups (Alexander et al. 2007b, 2010), but these variations are due, at least in large part, to processes that occurred on the asteroidal parent bodies of the chondrites (Alexander et al. 2007b; Alexander et al. 2010; Herd et al. 2011). In the meteorites (C1-2) that appear to have seen the most benign parent body conditions, the bulk composition of the IOM, normalized to $100 \mathrm{Cs}$, is $\sim \mathrm{C}_{100} \mathrm{H}_{70-79} \mathrm{~N}_{3-4} \mathrm{O}_{11-21} \mathrm{~S}_{1-5}$ (Alexander et al. 2007b). This is remarkably similar to the average composition of the comet Halley CHON particles $-\mathrm{C}_{100} \mathrm{H}_{80} \mathrm{~N}_{4} \mathrm{O}_{20} \mathrm{~S}_{2}$ - detected by the Vega 1 spacecraft (Kissel \& Krueger 1987).

Even amongst the C1-2 chondrites there is considerable elemental and isotopic variation in their IOM. The chondrites that harbor the apparently most primitive IOM are the CRs. Not only are the bulk $\mathrm{H}$ and $\mathrm{N}$ isotopic compositions of the IOM in CRs more anomalous than IOM from almost any other chondrite, but their IOM also contains small $(\leqslant 1 \mu \mathrm{m})$ isotopic hotspots that are more numerous and more extreme than in other chondrites (Busemann et al. 2006). The $\mathrm{D} / \mathrm{H}$ ratios of the hotspots can be up to $\sim 40$ times the terrestrial ratio, and ${ }^{15} \mathrm{~N} /{ }^{14} \mathrm{~N}$ ratios can be up to $\sim 4$ times the terrestrial ratio. The hotspot $\mathrm{D}$ and ${ }^{15} \mathrm{~N}$ enrichments overlap with the range of gas phase molecules in the ISM, but the two isotope systems are not correlated in any simple way. The bulk CR IOM 
Table 1. The abundances of insoluble and soluble organic compounds (in $\mu \mathrm{g} / \mathrm{g}$ or ppm) found in carbonaceous chondrites. For the CMs, all data are from the Murchison CM2 meteorite, unless otherwise noted (updated from Botta \& Bada 2002).

\begin{tabular}{|c|c|c|c|c|c|}
\hline $\mid$ & & CI & $\mathrm{CM}$ & | CR | & | Tag. Lake \\
\hline | & Matrix (vol\%) & | 100 & $\mid \sim 50$ & $|\sim 35 \quad|$ & $\sim 80$ \\
\hline | & IOM & $\mid \sim 20,000$ & $\mid \sim 10,000$ & $|\sim 5,000|$ & $\mid \sim 18,000$ \\
\hline | & Amino acids & $\sim 5^{a}$ & $14-71^{d}$ & $\left|1-250^{b}\right|$ & $0.04-5.6^{c}$ \\
\hline | & Aromatic hydrocarbons & | & $13^{d}$ & $16^{e} \quad \mid$ & l \\
\hline | & Aliphatic hydrocarbons & | & $\mid>35$ & | & | \\
\hline 1 & Monocarboxylic acids & I & | > $>300$ & $96^{e} \quad \mid$ & $165-448^{c}$ \\
\hline & droxy- and dicarboxylic acids & & | $14-15$ & $212^{e} \quad$ & I \\
\hline I & Purines and pyrimidines & I & | 1.3 & 1 & I \\
\hline I & Basic $\mathrm{N}$ heterocycles & | & I & | & | \\
\hline | & Amines & | & I & $103^{e} \mid$ & I \\
\hline 1 & Alcohols & I & 11 & । & | \\
\hline I & Aldehydes and Ketones & I & 27 & । & I \\
\hline I & Sulphonic acids & | & 68 & I & I \\
\hline & Phosphonic acids & | & 1 & I & | \\
\hline & Polyols & | & $\mid>8^{f}$ & | & | \\
\hline
\end{tabular}

Notes:

${ }^{a}$ Average for Orgueil and Ivuna (Ehrenfreund et al. 2001).

${ }^{b}$ Range from Martins et al. (2007).

${ }^{c}$ Herd et al. (2011).

${ }^{d}$ For Y-791198 (Naraoka et al. 1998).

${ }^{e}$ For GRA 95229 (Pizzarello et al. 2008).

${ }^{f}$ Lower limit of glyceric acid (Cooper et al. 2001).

isotopic compositions, and the range of hotspot compositions and sizes are very similar to those seen in the most primitive anhydrous chondritic IDPs (Messenger 2000; Aléon et al. 2003; Floss et al. 2006; Busemann et al. 2009), whose $\mathrm{H}$ and $\mathrm{N}$ isotopic compositions are dominated by those of their organics. The CR IOM H and $\mathrm{N}$ isotopic compositions are generally more anomalous than the compositions of organic particles identified in the Stardust mission samples (McKeegan et al. 2006; Matrajt et al. 2008). However, this could be because of modification during capture and, possibly, contamination.

The $\mathrm{H} / \mathrm{C}$ ratios of the primitive IOM are not unlike those of a fairly mature coal. Thus, it is not surprising that the IOM is fairly aromatic. Nuclear Magnetic Resonance (NMR) spectroscopy suggests that $\sim 50 \%$ of the $\mathrm{C}$ in $\mathrm{CR}$ IOM is in small PAHs (one to a few benzene rings), that these PAHs are heavily substituted and are cross-linked by short, highly branched aliphatic chains and O functionality (Cody \& Alexander 2005). IOM from CI and CM chondrites tend to be slightly more aromatic, but otherwise similar in structure to IOM in CRs (Gardinier et al. 2000; Cody, Alexander \& Tera 2002; Cody \& Alexander 2005; Cody et al. 2011). This confirms earlier work based on degradative chemistry (Hayatsu et al. 1977) and various pyrolysis techniques (Sephton, Pillinger \& Gilmour 1999; Sephton \& Gilmour 2001; Remusat et al. 2005; Sephton et al. 2005; Wang et al. 2005). The variations in aromatic $\mathrm{C}$ content are probably the result of parent body processes, and largely involved the conversion of aliphatic $\mathrm{C}$ to aromatic $\mathrm{C}$ and associated $\mathrm{H}$ loss (Herd et al. 2011). There also seems to be variable amounts of $\mathrm{H}$ 
isotope exchange and/or preferential D loss during this process. This suggests that the $\mathrm{D}$ is preferentially concentrated in the aliphatic fraction, an inference that appears to be confirmed by recent pyrolysis (Remusat et al. 2006) and NMR measurements (Wang et al. 2011). Simple heating of IOM above a few hundred degrees Celsius in an O-free atmosphere (pyrolysis) releases a lot of small PAHs and leaves a char. Heating of IOMlike material would be one mechanism for producing the PAHs that have been seen in protoplanetary disks. Sputtering of IOM-like material by energetic particles would also release PAHs.

The isotopic hotspots in IOM are not abundant enough to account for the bulk D and ${ }^{15} \mathrm{~N}$ enrichments. However, these hotspots are often associated with so-called globules or nanoglobules (Nakamura-Messenger et al. 2006). These globules are spherical to irregular in shape and often hollow (Nakamura et al. 2002; Garvie \& Buseck 2004; Garvie, Baumgardener \& Buseck 2008). Similar globules have now been found in IDPs and Stardust particles (Busemann et al. 2009; De Gregorio et al. 2010). Raman spectroscopy also suggests that the structure of the bulk of the organic material in the most primitive IDPs is similar to that of IOM from primitive chondrites, although on average it may be more disordered (Busemann et al. 2009). There is also a suggestion that the D in IDPs is largely associated with aliphatic material (Keller et al. 2004). However, based on IR spectra in the $3.4 \mu \mathrm{m} \mathrm{C}-\mathrm{H}$ stretch region, higher $\mathrm{CH}_{2} / \mathrm{CH}_{3}$ ratios in IDPs than in chondritic IOM suggests that the aliphatic material in IDPs tends to be shorter and/or more branched (Flynn et al. 2003; Keller et al. 2004; Muñoz Caro et al. 2006; Kebukawa, Alexander \& Cody 2011). Whether the greater disorder and higher $\mathrm{CH}_{2} / \mathrm{CH}_{3}$ ratios in IDPs is indigenous or was induced by irradiation in interplanetary space and atmospheric entry heating remains to be seen.

From the above, it is likely that there is a genetic relationship between the IOM and the organic matter in IDPs/comets, although the abundance of organic matter in IDPs and comets is much higher. The organic $\mathrm{C}$ contents are perhaps most usefully expressed in terms of the fractions of the bulk solar $\mathrm{C}$. Based on $\mathrm{Mg} / \mathrm{C}$ ratios, the percentage of solar $\mathrm{C}$ in CI chondrite IOM is $\sim 7 \%$, it is $\sim 30 \%$ on average in IDPs and $\geqslant 30 \%$ in comet Halley CHON particles (Alexander et al. 2007a). Thus, if there is a genetic relationship between IOM in chondrites and IDPs/comets, IOM-like material seems to have been widely distributed in the solar nebula but it formed or was better preserved in the outer Solar System. This along with the structural and isotopic properties of the IOM places considerable constraints on the various formation scenarios that have been proposed for the IOM.

One often invoked formation mechanism is Fischer-Tropsch-type (FTT) synthesis in the solar nebula (Hayatsu \& Anders 1981; Ferrante et al. 2000; Nuth, Hill \& Kletetschka 2000). Typically, FTT synthesis takes place on catalytic mineral surfaces, but there is no systematic association of the IOM with any mineral in meteorites (Remusat et al. 2010). Also, FTT synthesis would be most efficient in a narrow zone in the warm inner Solar System (Kress \& Tielens 2001). Hence it is unclear why IOM-like organics would be more abundant in the outer Solar System. Finally, FTT synthesis cannot explain the $\mathrm{D}$ and ${ }^{15} \mathrm{~N}$ enrichments in IOM. It has been pointed out that the conditions in the outer Solar System would have been quite similar to those in the protosolar molecular cloud. Therefore, the D enrichments in the IOM could have formed by reaction of IOM-like material with $\mathrm{H}_{2} \mathrm{D}^{+}$in the Solar System (Gourier et al. 2008; Remusat et al. 2009). However, if the conditions in the outer Solar System and the protosolar molecular cloud were so similar, this begs the question - what process destroyed the condensed organic C that appears to be so abundant in the diffuse and dense ISM, and then allowed organic $\mathrm{C}$ to reform so efficiently as IOM elsewhere in the Solar System? FTT synthesis also 
does not explain why there is a fairly constant ratio of circumstellar grains to IOM in chondrites (Alexander et al. 2007a,b).

Based largely, but not solely, on the isotopic composition of the IOM, an ISM origin for the IOM has been invoked (e.g., Robert \& Epstein 1982; Yang \& Epstein 1984; Alexander et al. 2007b). An ISM origin is strengthened by the similarity of the IR 3.4 $\mu \mathrm{m}$ C-H stretch adsorption feature of IOM and of carbonaceous dust in the diffuse ISM (Ehrenfreund et al. 1991; Pendleton et al. 1994). Some proposed structures for the diffuse ISM carbonaceous dust invoke small, highly substituted PAHs and short, highly branched aliphatic chains (Jones, Duley \& Williams 1990; Sandford et al. 1991; Dartois et al. 2005) and are remarkably similar to that of the IOM. On the other hand, Pendleton \& Allamandola (2002) concluded that the PAHs in the diffuse ISM dust were large and that the dust contains few $\mathrm{N}$ or $\mathrm{O}$ heteroatoms. Oxygen isotopes suggest that much of the $\mathrm{O}$ functionality in chondritic IOM is probably the result of parent body processes (Alexander et al. 2007b), and it is not clear whether the N content of IOM is inconsistent with the spectroscopic constraints for diffuse ISM dust. It is also important to point out that the spectra Pendleton \& Allamandola (2002) used is in fact a $600^{\circ} \mathrm{C}$ sublimate from IOM and is, therefore, not representative of the bulk IOM. Nevertheless, this does not explain the absence of large PAHs in IOM. Pendleton \& Allamandola (2002) concluded that the diffuse ISM dust probably formed in the outflows of C-stars. This too would be inconsistent with the IOM because the C-star outflows will be depleted in D and ${ }^{15} \mathrm{~N}$, and can have $\mathrm{C}$ isotopic compositions that are far from solar. Equally problematic to an interstellar origin for the IOM is that the $3.4 \mu \mathrm{m} \mathrm{C-H} \mathrm{stretch} \mathrm{feature} \mathrm{seen} \mathrm{in} \mathrm{the} \mathrm{diffuse}$ ISM dust and IOM is not seen in molecular clouds.

To overcome some of these problems, Alexander et al. (2008) suggested that in very cold molecular clouds cosmic ray irradiation of ice-coated diffuse ISM dust led to dehydrodgenation of aliphatic material and radiation damage of any large PAHs. Upon warming up, either as the dust enters a forming system or re-enters the diffuse ISM, the radicals in the damaged dust may react with radicals in the ice (e.g., $\mathrm{H}, \mathrm{OH}, \mathrm{NH}_{2}, \mathrm{CH}_{3}$, etc.) rather than reform their original bonds. Since the ice is likely to be D- and ${ }^{15} \mathrm{~N}$-rich, this process could explain both the heteroatom content, and D and ${ }^{15} \mathrm{~N}$ enrichments in the IOM. Dust re-entering the diffuse ISM would have to loose its O functionality and remake any large PAHs, processes that would presumably have to be driven by the intense UV radiation field that exists there. This model should be testable experimentally. However, it does not explain the solar $\mathrm{C}$ isotopic composition of the IOM or the formation of globules. The $\mathrm{C}$ isotopes might be explained if in fact the typical lifetime of carbonaceous dust in the ISM is short compared to the production rate by C-stars (Serra Díaz-Cano \& Jones 2008). In this case, there must be an efficient mechanism for making the dust in the ISM, and the bulk $\mathrm{C}$ isotopic composition of the dust that forms in the ISM is likely to have been close to the bulk isotopic composition of the ISM (i.e., solar) at that time. Nakamura-Messenger et al. (2006) suggested that globules could have formed by irradiation of ISM ices, but in their mechanism the wall thicknesses of the globules would reflect the penetration depth of the radiation (10s to a few hundred nanometers), which is too little for cosmic rays and probably requires unreasonably high $\mathrm{C}$ contents for the ice.

A quite different scenario for IOM formation is envisaged by Cody et al. $(2008 ; 2011)$. They suggest that the IOM formed by polymerization of formaldehyde when accreted ice melted as meteorite parent bodies warmed due to the decay of short-lived radionuclides. This process naturally produces globule-like objects and can produce material with NMR spectra that resembles IOM on heating. If the ice formed in the ISM or outer Solar System, it and the formaldehyde it contained would probably be D-rich and contain ${ }^{15} \mathrm{~N}$-rich 
N-bearing species that could be incorporated into the IOM. This model faces two hurdles. The first is whether there would have been enough formaldehyde in the ices to explain the abundances of IOM or IOM-like material in chondrites and, particularly, in IDPs and comets. This is especially problematic given that polymerization is an inherently inefficient process. The second is whether liquid water was present in cometary bodies, and whether the kinetics of the transformation of the initial polymer to more IOM-like material could take place at reasonable temperatures and over reasonable timescales for either comets or chondrites. The latter is experimentally testable, and the former has gained at least some recent support from the Stardust mission (Berger et al. 2011).

Ultimately, whether the organics in chondrites, IDPs and comets are solar or interstellar in origin may be determined not by the properties of the organics themselves, but rather by the abundance and origin of amorphous silicate material in IDPs and comets (Alexander et al. 2007a). Silicates in the ISM are essentially entirely amorphous. In IDPs and comets, on the other hand, crystalline silicates are a major component and must have formed at temperatures $(>1000 \mathrm{~K})$ that would have destroyed any interstellar organics. There is amorphous silicate material in primitive chondrites and IDPs, but there is an ongoing debate about whether the bulk of it is interstellar or not. Isotope analyses show that a few percent of the amorphous material in IDPs is circumstellar in origin. Given the short lifetime of circumstellar dust in the ISM, it seems likely that a significant fraction of the remaining amorphous material must be interstellar. Unfortunately, it is difficult to prove an interstellar origin and energetic Solar System processes (e.g., irradiation, impacts and condensation from a hot gas) can produce material with similar properties. Stardust samples do contain amorphous material, but it is hard to distinguish between indigenous material and that produced during capture. Comets are also known to contain amorphous silicates, but the results of the Deep Impact mission show that the grains being emitted by comets may not be representative of the bulk (Lisse et al. 2007). Nevertheless, one can roughly explain the abundances of organic C in IDPs and comet Halley if: (1) one assumes that all amorphous silicates in IDPs are interstellar and that $\sim 50 \%$ of comet Halley silicates are amorphous and interstellar, and (2) adopt the range of abundances for carbonaceous dust and PAHs in the ISM inferred by Zubko, Dwek \& Arendt (2004). Obviously, if a large fraction of the amorphous silicates proves to be solar in origin, then a proportional fraction of the organics must also be solar.

\subsection{Soluble Organic Matter}

The SOM in primitive chondrites is a complex suite of soluble compounds. The best characterized of these are summarized in Table 1. However, it seems likely that the bulk of the SOM has yet to identified (Schmitt-Kopplin et al. 2010). This poorly characterized material is composed of $>46,000$ compounds with masses of up to at least 1000 daltons and an approximate average composition of $\mathrm{C}_{100} \mathrm{H}_{155} \mathrm{~N}_{3} \mathrm{O}_{20} \mathrm{~S}_{3}$ that resembles an IOM composition but with roughly twice the $\mathrm{H}$ content. Thus, it seems likely that the IOM and the uncharacterized SOM are related, but the uncharacterized SOM is presumably more aliphatic. Even within the classes of well-characterized SOM compounds there is tremendous structural diversity, although abundances tend to decrease significantly with increasing $\mathrm{C}$ number and extent of branching. The abundances of volatile compounds could also have been modified during the residence of the meteorites on Earth. Until we have a better understanding of the nature of the uncharacterized material, the work on the known soluble components probably presents a rather distorted picture of the origins of the SOM. Nevertheless, important features of the well-characterized SOM are briefly discussed below. 
Even more than the IOM, the soluble organics are susceptible to modification (isotopic exchange, synthesis and destruction) during parent body processing. For instance, amino acid abundances decline dramatically within a chondrite group with increasing degree of alteration (Martins et al. 2007; Martins \& Sephton 2009; Glavin et al. 2010; Herd et al. 2011). In the least altered chondrites, the amino acids are racemic within error. Surprisingly, the development of $\mathrm{L}$ enantiomeric excesses in amino acids like isovaline that do not easily racemize accompanies this decrease in abundance with increasing alteration (Glavin et al. 2010). Apparently, aqueous alteration either produced the enantiomeric excesses or amplified small excesses that already existed. The mechanism that produced or amplified these enantiomeric excesses is unknown, but one intriguing possibility is that it involves the IOM, which is known to possess chiral centers (Kawasaki et al. 2006).

The amino acids themselves are thought to be products of Strecker synthesis, the reaction of ketones and aldehydes with $\mathrm{NH}_{3}$ and $\mathrm{HCN}$. Presumably amino acid synthesis generally took place very early on in the alteration of the meteorites, but in the case of the Tagish Lake meteorite there may have been a second episodes of synthesis after alteration was fairly advanced (Herd et al. 2011). The fact that the relatively volatile $\mathrm{NH}_{3}$ and $\mathrm{HCN}$ were in the ices that were accreted by the chondrites again suggests a link with comets. The $\mathrm{HCN}$ in comets is enriched in ${ }^{15} \mathrm{~N}$ by roughly a factor of two over terrestrial (Shinnaka et al. 2011). The $\mathrm{N}$ isotopic composition in cometary $\mathrm{NH}_{3}$ has not been measured, but in the ISM it is depleted in ${ }^{15} \mathrm{~N}$ relative to terrestrial (Lis et al. 2010). The amino acids and $\mathrm{NH}_{3}$ in two relatively unaltered CRs (GRA 95229 and LAP 02342) are only enriched in ${ }^{15} \mathrm{~N}$ by about $10 \%$ over terrestrial (Pizzarello \& Holmes 2009). In the Strecker synthesis, it is the $\mathrm{N}$ from the $\mathrm{NH}_{3}$, not the $\mathrm{HCN}$, that is inherited by the amino acid. The $\mathrm{N}$ from the $\mathrm{HCN}$ is converted into $\mathrm{NH}_{3}$, which may eventually be incorporated into amino acids but only after significant dilution by pre-existing $\mathrm{NH}_{3}$, which may explain the modest ${ }^{15} \mathrm{~N}$ enrichments in $\mathrm{NH}_{3}$ and amino acids.

The $\mathrm{H}$ isotopic compositions of amino acids have also been measured in one relatively unaltered CR (GRA 95229), and two more altered CMs (Murchison and Murray) (Pizzarello \& Huang 2005; Pizzarello, Huang \& Alexandre 2008). In a given meteorite, the D enrichment in straight chain amino acids tends to increase with increasing $\mathrm{C}$ number, and branched amino acids are more enriched than both the straight chain ones and the bulk IOM from the same meteorites. The amino acids in the more altered CMs are systematically less D-rich than in the less altered CR. The isotopic compositions of the putative precursor aldehydes and ketones are unknown, so it cannot be ruled out that some of the isotopic variations in the amino acids were inherited. Nevertheless, the patterns of isotopic variations are suggestive of isotopic exchange with the aqueous fluid during or after synthesis. Whatever the cause of the variations, the $\mathrm{D}$ enrichments (up to 8 times terrestrial) indicate that, like the IOM, the amino acids or their precursors formed in cold environments, either in the outer Solar System or the ISM. However, the C isotopic compositions of the amino acids are distinctly (>3-5\%) more ${ }^{13} \mathrm{C}$-rich than the IOM and are closer to those of the carbonate minerals that are present in aqueously altered chondrites (Engel, Macko \& Silfer 1990; Pizzarello, Huang \& Fuller 2004; Martins et al. 2007).

In the more altered CM Murchison, carboxylic acids rather than amino acids are the most abundant of the well-characterized SOM. In the Tagish Lake meteorite, in which a range of alteration is recorded, carboxylic acid abundances do seem to be a function of the extent of aqueous alteration (Herd et al. 2011). As with the amino acids, the $\mathrm{H}$ isotopic compositions of the straight chain carboxylic acids vary with $\mathrm{C}$ number, and the branched carboxylic acids are more D-rich (Huang et al. 2005; Herd et al. 2011). Again, the $\mathrm{D}$ enrichments of the carboxylic acids indicate that they or their precursors have a 
cold chemistry heritage. It is tempting to invoke a simple conversion of amino acids to carboxylic acids, but the $\mathrm{C}$ isotopic compositions of the carboxylic acids are inconsistent with this. The ${ }^{13} \mathrm{C}$ abundances of the straight chain carboxylic acids in Murchison decrease with increasing $\mathrm{C}$ number down to isotopic compositions that resemble those of the IOM (Huang et al. 2005). Based on both the $\mathrm{H}$ and $\mathrm{C}$ isotopic compositions, it is possible that the carboxylic acids in Murchison and Murray are related to or were derived from the aliphatic component of the IOM (Huang, Alexandre \& Wang 2007; Herd et al. 2011). The variation in $\mathrm{C}$ isotopic composition with chain length seen in Murchison and one Tagish Lake sample could reflect isotopic exchange with dissolved $\mathrm{CO}_{2}$ with a composition like that of the carbonate minerals (Herd et al. 2011).

Comets contain simple alkanes and alkenes (Mumma \& Charnley 2011). The same is true at least of the CM Murchison (Yuen et al. 1984). However, Murchison also contains $\mathrm{CO}$ and $\mathrm{CO}_{2}$, several well-known mineral catalysts of FTT synthesis and would have generated considerable amounts of $\mathrm{H}_{2}$ during alteration. Thus, at this stage it should not be ruled out that the simple hydrocarbons, as well as other components of the SOM are the products of FTT synthesis during alteration rather than being primordial.

\section{Water in Chondrites}

The O isotopic compositions of inner Solar System objects all appear to be more ${ }^{16} \mathrm{O}$ poor than the composition of the Sun. UV self-shielding during the photodissociation of CO in the outer Solar System or near the surface of a molecular cloud can produce

${ }^{16} \mathrm{O}$-poor water ice. Hence, the favored explanation for the $\mathrm{O}$ isotopic composition of the terrestrial planet region is that there was a massive influx of outer Solar System and/or interstellar water ice into the inner Solar System (Yurimoto \& Kuramoto 2004; Lyons \& Young 2005). This ice would have been accompanied by organic material and other primitive materials. Chondrites, except probably the enstatite chondrites, seem to have accreted water ice, indicating that they formed beyond the snowline. When they formed, all chondrites also contained circumstellar grains and, as argued above, organics that are genetically linked to organics in IDPs/comets. In comets, $\mathrm{NH}_{3}$ and $\mathrm{HCN}$ are major volatile components. The fact that these relatively volatile compounds are needed for making the amino acids in chondrites strengthens the comet-chondrite connection.

The $\mathrm{D} / \mathrm{H}$ ratio in water ice is expected to increase with radial distance from the Sun as temperatures and pressures decrease, and conditions approached those in molecular clouds. In most, but not all, comets the $\mathrm{H}$ isotopic compositions of the water are enriched in D by roughly a factor of 2 relative to terrestrial values (Mumma \& Charnley 2011). Given the apparent relationship between chondritic and IDP/comet organics, one might expect that, as with comets, chondritic water would be similarly enriched in D.

The $\mathrm{H}$ isotopic composition of chondritic water is difficult to measure directly. This is because it is now primarily incorporated into hydrous silicates as $\mathrm{OH}^{-}$. Heating of the meteorites does release the water, but also releases $\mathrm{H}$ from organics that will exchange/mix with the $\mathrm{H}$ from the hydrous silicates. The $\mathrm{H}$ isotopes of hydrated silicates can be measured at small scales $(<30 \mu \mathrm{m})$ in materials that are free or organics or using techniques that preferentially measure $\mathrm{H}$ in hydrous minerals (Deloule, Robert \& Doukhan 1998; Grossman et al. 2002; Bonal et al. 2011). The average H isotopic compositions of the water in some chondrites have also been estimated by subtracting the known organic materials that have been isolated from them (Robert 2002; Alexander et al. 2010).

From the range of $\mathrm{D}$ enrichments recorded in these kinds of measurements and estimates, it has been argued that the water in chondrites is composed of two components, 
one that is much more D-rich than comets and may be interstellar, and another that formed at relatively high temperatures in the inner Solar System so that it has a H isotopic composition that is only slightly more D-rich than the solar composition (Deloule et al. 1998; Robert 2001; Robert 2002). More recently it has been suggested that the $\mathrm{H}$ isotopic composition of water in chondrites at all spatial scales has been enriched in D to varying degrees by two parent body processes (Alexander et al. 2010; Alexander et al. 2011; Bonal et al. 2011): (1) the oxidation of Fe by water and the consequent loss of $\mathrm{H}_{2}$, and (2) by isotopic exchange with D-rich organics. If correct, none of the chondrites accreted water ice that was as D-rich as in most comets. The exact $\mathrm{H}$ isotopic compositions at the time of accretion are still uncertain, but in the CM and CI chondrites their $\mathrm{D} / \mathrm{H}$ ratios were probably significantly below the terrestrial composition. Presumably the CM and CI water formed or re-equilibrated at smaller radial distances than typical cometary ices. How this can be reconciled with the presence of IDP/comet-like organics in chondrites, as well as $\mathrm{NH}_{3}$ and $\mathrm{HCN}$ in the chondritic ices, remains to be seen.

While it is still not clear when the Earth and the other terrestrial planets accreted their volatiles, if it was at a late stage it would have been after parent body processes on the chondrites' parental asteroids had ceased. Thus, if the chondrites were the sources of the volatiles, their present bulk compositions (water and organics) should reflect what would have been accreted. Not only the $\mathrm{H}$ isotopes constrain the likely sources of the volatiles, but also the relative abundances and isotopic compositions of $\mathrm{N}$ and the noble gases. At present, no single chondrite group can explain all of these constraints.

\section{Summary and Implications}

Chondritic meteorites preserve a record of materials that were present in the first few million years of solar nebula evolution. These materials include demonstrably presolar circumstellar grains that were inherited from the protosolar molecular cloud. Most, if not all, chondrites also accreted organic matter when they formed, but generally this material has been heavily modified in the asteroidal parent bodies. In three chondrite groups (CI, $\mathrm{CM}$, and $\mathrm{CR}$ ) the organic matter has been reasonably well preserved, although it has been modification to varying degrees by hydrothermal alteration. The organic matter in the CI-CM-CR chondrites is broadly divided into solvent soluble and insoluble fractions.

The insoluble fraction (IOM) is a structurally complex, macromolecular material composed of small aromatic moieties that are cross-linked by short, highly branched aliphatic chains. It has a bulk elemental composition that is similar to the average of comet Halley CHON particles, and is dispersed in chondrite matrices as particles that are typically $<1$ $\mu \mathrm{m}$ across. In bulk, the insoluble material is significantly enriched in $\mathrm{D}$ and ${ }^{15} \mathrm{~N}$. Even more extreme isotopic enrichments can be found in localized (usually $<1 \mu \mathrm{m}$ across) hotspots, some of which are associated with so-called globules - spherical to irregular organic particles that are often hollow.

Similar globules, isotopic hotspots and/or bulk isotopic enrichments are found in the organic matter of chondritic interplanetary dust particles and comet $81 \mathrm{P} /$ Wild 2 samples. Thus, there is a genetic link between objects that formed between $\sim 3 \mathrm{AU}$ and $\sim 30 \mathrm{AU}$ from the Sun. The enrichments attest to formation in very cold environments, either in the outer Solar System or the protosolar molecular cloud. There are many properties of this organic material that are consistent with an interstellar origin, e.g., hotspot isotopic compositions overlap with those of gas-phase molecules in molecular clouds, a $3.4 \mu \mathrm{m}$ IR adsorption feature resembles that of diffuse ISM dust and abundances relative to circumstellar grains are similar in all chondrites. However, at present a Solar System origin cannot be ruled out. Since the early Solar System does not appear to have been 
particularly unusual and this organic material was so widespread in the solar nebula, presumably similar material is an important component of most protoplanetary disks. Heating or sputtering of this material would be a source of PAHs in disks.

The solvent-extractable organic material (SOM) is a complex suite of compounds. Many of these compounds show almost complete structural diversity for a given carbon number, and also have large enrichments in $\mathrm{D}$ and, if they contain $\mathrm{N}$, in ${ }^{15} \mathrm{~N}$. The amino acids are thought to have formed by reaction of aldehydes and ketones with $\mathrm{NH}_{3}$ and $\mathrm{HCN}$ very early in the aqueous alteration of the CI-CM-CRs. The fact that volatile $\mathrm{NH}_{3}$ and HCN were accreted, presumably in ice, strengthens the chondrite-comet connection. Some amino acids in CI-CM-CR chondrites do not readily racemize exhibit 0-20\% Lenantiomeric excesses. The L-enantiomeric excesses seem to increase with increasing hydrothermal alteration and decreasing amino acid abundances. Thus, the L-enantiomeric excesses appear to be the product of the alteration or the alteration has amplified small excesses that were inherited at the time of accretion.

Despite the links between chondrites and comets suggested by the organic matter, unlike most comets the water in chondrites, when it was accreted, had $\mathrm{D} / \mathrm{H}$ ratios that were similar to or depleted relative to Earth. The water in chondrites seems to have formed or isotopically re-equilibrated with $\mathrm{H}_{2}$ at the higher temperatures of the inner Solar System. How this can be reconciled with the preservation of primitive organics and accretion of volatiles like $\mathrm{NH}_{3}$ and $\mathrm{HCN}$ remains to be seen.

\section{References}

Aléon, J., Robert, F., Chaussidon, M., \& Marty, B. 2003, Geochim. Cosmochim. Acta, 67, 3773

Alexander, C. M. O'D. 2009, in: F. Boulanger, C. Joblin, A. Jones, \& S. Madden (eds.) Interstellar dust from astronomical observations to fundamental studies, (European Astronom. Soc. Publ. Series), p. 75

Alexander, C. M. O'D., Boss, A. P., Keller, L. D., Nuth, I., J. A., \& Weinberger, A. 2007a, in: B. Reipurth, D. Jewitt, \& K. Keil (eds.) Protostars and Planets V, (Tucson: University of Arizona Press), p. 801

Alexander, C. M. O’D., Cody, G. D., Fogel, M., \& Yabuta, H. 2008, in: S. Kwok, \& S. A. Sandford (eds.) Organic Matter in Space (Hong Kong: Cambridge University Press), p. 293

Alexander, C. M. O'D., Fogel, M., Yabuta, H., \& Cody, G. D. 2007b, Geochim. Cosmochim. Acta, 71, 4380

Alexander, C. M. O'D., Howard, K. T., Bowden, R., Fogel, M., \& Bonal, L. 2011, Lunar Planet. Sci., 42, \#1869

Alexander, C. M. O'D., Newsome, S. N., Fogel, M. L., Nittler, L. R., Busemann, H., \& Cody, G. D. 2010, Geochim. Cosmochim. Acta, 74, 4417

Berger, E. L., Zega, T. J., Keller, L. P., \& Lauretta, D. S. 2011, Geochim. Cosmochim. Acta, 75, 3501

Bonal, L., Alexander, C. M. O'D., Huss, G. R., \& Nagashima, K. 2011, Lunar Planet. Sci., 42, \#1287

Botta, O. \& Bada, J. L. 2002, Surveys in Geophysics, 23, 411

Burbine, T. H., McCoy, T. J., Meibom, A., Gladman, B., \& Kiel, K. 2002, in Asteroids III, eds. W. F. Bottke, Jr., A. Cellino, P. Paolicchi, \& R. P. Binzel (Tuscon: University of Arizona Press), 653

Busemann, H., et al. 2009, Earth Planet. Sci. Lett., 288, 44

Busemann, H., Young, A. F., Alexander, C. M. O'D., Hoppe, P., Mukhopadhyay, S., \& Nittler, L. R. 2006, Science, 314, 727

Cody, G. D. \& Alexander, C. M. O'D. 2005, Geochim. Cosmochim. Acta, 69, 1085

Cody, G. D., Alexander, C. M. O’D., Kilcoyne, A. L. D., \& Yabuta, H. 2008, in: S. Kwok, \& S. A. Sandford (eds.) Organic matter in space, (Hong Kong: Cambridge University Press), p. 277 
Cody, G. D., Alexander, C. M. O'D., \& Tera, F. 2002, Geochim. Cosmochim. Acta, 66, 1851

Cody, G. D., Heying, E., Alexander, C. M. O’D., Nittler, L. R., Kilcoyne, A. L. D., Sandford, S. A., \& Stroud, R. M. 2011, Proc. Nat. Acad. Sci., 108,

Cooper, G., Kimmich, N., Belisle, W., Sarinana, J., Brabham, K., \& Garrel, L., 2001, Nature, 414,879

Dartois, E., Muñoz Caro, G. M., Deboffle, D., Montagnac, G., \& d'Hendecourt, L. 2005, Astron. Astrophys., 432, 895

De Gregorio, B. T., Stroud, R. M., Nittler, L. R., Alexander, C. M. O’D., Kilcoyne, A. L. D., \& Zega, T. J. 2010, Geochim. Cosmochim. Acta, 74, 4454

Deloule, E., Robert, F., \& Doukhan, J. C. 1998, Geochim. Cosmochim. Acta, 62, 3367

Dermott, S. F., Durda, D. D., Grogan, K., \& Kehoe, T. J. J. 2002, in: W. F. Bottke, Jr., A. Cellino, P. Paolicchi, \& R. P. Binzel (eds.) Asteroids III, (Tucson: University of Arizona Press), p. 423

Duprat, J., et al. 2010, Science, 328, 742

Ehrenfreund, P., Glavin, D. P., Botta, O., Cooper, G., \& Bada, J. L. 2001, Proc. Nat. Acad. Sci., 98, 2138

Ehrenfreund, P., Robert, F., d'Hendencourt, L., \& Behar, F. 1991, Astron. Astrophys., 252, 712

Engel, M. H., Macko, S. A., \& Silfer, J. A. 1990, Nature, 348, 47

Ferrante, R. F., Moore, M. H., Nuth, J. A., \& Smith, T. 2000, Icarus, 145, 297

Floss, C., Stadermann, F. J., Bradley, J. P., Dai, Z. R., Bajt, S., Graham, G. A., \& Lea, A. S. 2006, Geochim. Cosmochim. Acta, 70, 2371

Flynn, G. J., Keller, L. P., Feser, M., Wirick, S., \& Jacobsen, C. 2003, Geochim. Cosmochim. Acta, 67, 4791

Gardinier, A., Derenne, S., Robert, F., Behar, F., Largeau, C., \& Maquet, J. 2000, Earth Planet. Sci. Lett., 184, 9

Garvie, L. A. J., Baumgardener, G., \& Buseck, P. R. 2008, Meteor. Planet. Sci., 43, 899

Garvie, L. A. J. \& Buseck, P. R. 2004, Earth Planet. Sci. Lett., 224, 431

Gilmour, I. 2003, in: A. M. Davis (ed.) Meteorites, Comets and Planets, (Oxford: ElsevierPergamon), p. 269

Glavin, D. P., Callahan, M. P., Dworkin, J. P., \& Elsila, J. E. 2010, Meteor. Planet. Sci., 45, 1948

Gourier, D., Robert, F., Delpoux, O., Binet, L., Vezin, H., Moissette, A., \& Derenne, S. 2008, Geochim. Cosmochim. Acta, 72, 1914

Gradie, J. C., Chapman, C. R., \& Tedesco, E. F. 1989, in: R. P. Binzel, T. Gehrels, \& M. S. Matthews (eds.) Asteroids II, (Tucson: Univ. Arizona Press), p. 316

Grossman, J. N., Alexander, C. M. O'D., Wang, J., \& Brearley, A. J. 2002, Meteor. Planet. Sci., 37,49

Hayatsu, R. \& Anders, E. 1981, in: F. L. Boschke (ed.) Cosmo- and Geochemistry (Berlin: Springer-Verlag), p. 1

Hayatsu, R., Matsuoka, S., Scott, R. G., Studier, M. H., \& Anders, E. 1977, Geochim. Cosmochim. Acta, 41, 1325

Herd, C. D. K., et al. 2011, Science, 332, 1304

Huang, Y., Alexandre, M. R., \& Wang, Y. 2007, Earth Planet. Sci. Lett., 259, 517

Huang, Y., Wang, Y., Alexandre, M. R., Lee, T., Rose-Petruck, C., Fuller, M., \& Pizzarello, S. 2005, Geochim. Cosmochim. Acta, 69, 1073

Jones, A. P., Duley, W. W., \& Williams, D. A. 1990, QJRAS, 31, 567

Kawasaki, T., Hatase, K., Fujii, Y., Jo, K., Soai, K., \& Pizzarello, S. 2006, Geochim. Cosmochim. Acta, 70, 5395

Kebukawa, Y., Alexander, C. M. O’D., \& Cody, G. D. 2011, Geochim. Cosmochim. Acta, 75, 3530

Keller, L. P., Messenger, S., Flynn, G. J., Clemett, S., Wirick, S., \& Jacobsen, C. 2004, Geochim. Cosmochim. Acta, 68, 2577

Kissel, J. \& Krueger, F. R. 1987, Nature, 326, 755

Kress, M. E. \& Tielens, A. G. G. M. 2001, Meteor. Planet. Sci., 36, 75

Krot, A. N., Keil, K., Goodrich, C. A., Scott, E. R. D., \& Weisberg, M. K. 2003, in: A. M. Davis (ed.) Meteorites, comets and planets, (Oxford: Elsevier-Pergamon), p. 83 
Lis, D. C., Wootten, A., Gerin, M., \& Roueff, E. 2010, ApJ, 710, L49

Lisse, C. M., Kraemer, K. E., Nuth, J. A., Li, A., \& Joswiak, D. 2007, Icarus, 191, 223

Lodders, K. 2003, ApJ, 591, 1220

Lyons, J. R. \& Young, E. D. 2005, Nature, 435, 317

Martins, Z., Alexander, C. M. O’D., Orzechowska, G. E., Fogel, M. L., \& Ehrenfreund, P. 2007, Meteor. Planet. Sci., 42, 2125

Martins, Z. \& Sephton, M. 2009, in: A. B. Hughes (ed.) Origins and synthesis of amino acids, (Weinheim: Wiley-VCH), p. 3

Matrajt, G., et al. 2008, Meteor. Planet. Sci., 43, 315

McKeegan, K. D., et al. 2006, Science, 314, 1724

Messenger, S. 2000, Nature, 404, 968

Morbidelli, A., Bottke, W. F., Jr., Froeschle, C., \& Michel, P. 2002, in: W. F. Bottke, Jr., A. Cellino, P. Paolicchi, \& R. P. Binzel (eds.) Asteroids III, (Tucson: University of Arizona Press), p. 409

Mumma, M. J. \& Charnley, S. B. 2011, ARAA, 49, 471

Muñoz Caro, G. M., et al. 2006, Astron. Astrophys., 459, 147

Nakamura, K., Zolensky, M. E., Tomita, S., Nakashima, S., \& Tomeoka, K. 2002, Int. J. Astrobiol., 1,179

Nakamura-Messenger, K., Messenger, S., Keller, L. P., Clemett, S. J., \& Zolensky, M. E. 2006, Science, 314, 1439

Naraoka, H., Shimoyama, A., Komiya, M., Yamamoto, H., \& Harada, K. 1988, Chem. Lett., 17, 831

Nesvorny, D., Jenniskens, P., Levison, H. F., Bottke, W. F., Vokrouhlicky, D., \& Gounelle, M. 2010, ApJ, 713, 816

Nittler, L. R. 2003, Earth Planet. Sci. Lett., 209, 259

Nuth, J. A., Hill, H. G. M., \& Kletetschka, G. 2000, Nature, 406, 275

Pendleton, Y. J. \& Allamandola, L. J. 2002, ApJS, 138, 75

Pendleton, Y. J., Sandford, S. A., Allamandola, L. J., Tielens, A. G. G. M., \& Sellgren, K. 1994, ApJ, 437, 683

Pizzarello, S., Cooper, G. W., \& Flynn, G. J. 2006, in: D. S. Lauretta, \& H. Y. McSween Jr. (eds.) Meteorites and the Early Solar System II, (Tucson: University of Arizona Press), p. 625

Pizzarello, S. \& Holmes, W. 2009, Geochim. Cosmochim. Acta, 73, 2150

Pizzarello, S., Huang, Y., \& and Alexandre, M. R. 2008, Proc. Nat. Acad. Sci. 105, 3700

Pizzarello, S. \& Huang, Y. 2005, Geochim. Cosmochim. Acta, 69, 599

Pizzarello, S., Huang, Y., \& Alexandre, M. R. 2008, Proc. Nat. Acad. Sci., 105, 3700

Pizzarello, S., Huang, Y., \& Fuller, M. 2004, Geochim. Cosmochim. Acta, 68, 4963

Remusat, L., Derenne, S., Robert, F., \& Knicker, H. 2005, Geochim. Cosmochim. Acta, 69, 3919

Remusat, L., Guan, Y., Wang, Y., \& Eiler, J. M. 2010, ApJ, 713, 1048

Remusat, L., Palhol, F., Robert, F., Derenne, S., \& France-Lanord, C. 2006, Earth Planet. Sci. Lett., 243, 15

Remusat, L., et al. 2009, ApJ, 698, 2087

Robert, F. 2001, Science, 293, 1056

Robert, F. 2002, Planet. Space Sci., 50, 1227

Robert, F. \& Epstein, S. 1982, Geochim. Cosmochim. Acta, 46, 81

Sandford, S. A., Allamandola, L. J., Tielens, A. G. G. M., Sellgren, K., Tapia, M., \& Pendleton, Y. J. 1991, ApJ, 371, 607

Schmitt-Kopplin, P., et al. 2010, Proc. Nat. Acad. Sci., 107, 2763

Scott, E. R. D. \& Krot, A. N. 2003, in: A. M. Davis (ed.) Meteorites, Comets and Planets, (Oxford: Elsevier-Pergamon), p. 143

Sephton, M. A. 2002, Nat. Prod. Rep., 19, 292

Sephton, M. A. 2005, Phil. Trans. Roy. Soc. A, 363, 2729

Sephton, M. A. \& Gilmour, I. 2001, Planet. Space Sci., 49, 465

Sephton, M. A., Love, G. D., Meredith, W., Snape, C. E., Sun, C.-G., \& Watson, J. S. 2005, Planet. Space Sci., 53, 1280 
Sephton, M. A., Pillinger, C. T., \& Gilmour, I. 1999, Planet. Space Sci., 47, 181

Serra Díaz-Cano, L. \& Jones, A. P. 2008, Astron. Astrophys., 492, 127

Shinnaka, Y., Kawakita, H., Kobayashi, H., Jehin, E. 1., Manfroid, J., Hutsemékers, D., \& Arpigny, C. 2011, ApJ, 729, 81

Wang, Y., Huang, Y., Alexander, C. M. O'D., Fogel, M., \& Cody, G. 2005, Geochim. Cosmochim. Acta, 69, 3711

Wang, Y., Kebukawa, Y., Cody, G. D., \& Alexander, C. M. O’D. 2011, Lunar Planet. Sci., 42, $\# 2380$

Weisberg, M. K., McCoy, T. J., \& Krot, A. N. 2006, in: D. S. Lauretta, \& H. Y. McSween, Jr. (ed.) Meteorites and the Early Solar System II, (Tucson: University of Arizona Press), 19

Yang, J. \& Epstein, S. 1984, Nature, 311, 544

Yuen, G., Blair, N., Des Marais, D. J., \& Chang, S. 1984, Nature, 307, 252

Yurimoto, H. \& Kuramoto, K. 2004, Science, 305, 1763

Zubko, V., Dwek, E., \& Arendt, R. G. 2004, ApJS, 152, 211

\section{Discussion}

IRVING: How are the enantiomeric excesses in some SOM compounds produced?

Alexander: At this point, it is a complete mystery, but the answer obviously has tremendous astrobiological implications.

SARRE: It somewhat surprises me that PAHs are not seen in comets. Could it be that they are tied up in IOM-like material?

AlEXANDER: In meteorites there are small amounts of free PAHs, but the vast majority are tied up in IOM. So if, as seems likely, the organics in chondrites are related to those in comets, tying up most of the PAHs in IOM-like material seems a likely explanation for why PAHs are not seen in comets.

ELLINGER: Do you have structural information about the position of the D in the IOM and SOM?

AlexAnDER: In the IOM several lines of evidence suggest that the D is primarily associated with the aliphatic material. For the individual SOM compounds, there is not information about whether specific functional groups are more deuterated than others. 